\title{
Solitary Wave Solutions to Certain Nonlinear Evolution Equations by Rational Sine -Cosine Function Method
}

\author{
S. Subhaschandra Singh, \\ Department of Physics, Dhanamanjuri College of Science, Imphal - 795 001, Manipur, India.
}

\begin{abstract}
In this paper, soliton solutions to four Nonlinear Evolution Equations (NLEEs) namely Boussinesq Equation (BE), Gardner Equation (GE), (coupled) Generalized Boussinesq-Burgers Equations (GBBE) and Mikhailov-Shabat system of Equations (MSE) are obtained by the Rational Sine-Cosine Method. It has been demonstrated that the method is a convenient and effective one for solving a wide class of NLEEs encountered in various areas of Nonlinear Physical Sciences.
\end{abstract}

PACS NOS.:02.30Jr, 5.45Yv, 87.10Ed.

AMS Mathematics Subject Classification(2010) Nos.:35C08, 35L05, 35Q51, $37 \mathrm{~K} 40$.

Key words:Soliton, NLEEs, BE, GE, GBBE, MSE, Rational Sine-Cosine Method, Nonlinear Physical Sciences, Plasma Physics, QFT.

\section{Introduction}

It is well known that the studies of Nonlinear Evolution Equations (NLEEs) [which are nothing but Nonlinear Partial Differential Equations (NLPDEs)] have attracted the attention of many researchers because of their wide range applications in various fields of Science, Social Science and Engineering, especially inFluid Mechanics, Material Science, Plasma Physics, Nonlinear Optics, Geophysics, Biophysics, Neurophysics, Fiber Optics, Chemical Physics, Biomathematics, Ecology, Economics, Demography, Population Dynamics, etc. Nowadays, research in Physics is devoting much attention to NLEEs appearing in various fields of Science and Engineering. To understand the Nonlinear Phenomena better and to apply them in real life situations, it is important to find the exact solutions of the relevant NLEEs. Quite a number of different methods for obtaining exact solutions of NLEEs have been established so far. In this paper, we apply the Rational Sine-Cosine Method [1] for obtaining Soliton solutions toBE[2-7 ], GE[8 - 12],GBBE[13] and MSE [14].By this method, one can solve many other NLEEs arose in Nonlinear Science and Engineering.

\section{Outlines of Rational Sine-Cosine Method}

Let a given NLEE be expressible in the form

$$
F\left(u, u_{t}, u_{t t}, u_{x}, u_{x x}, u_{x t}, u_{x x t}, u_{x t t}, \ldots\right)=0 \text {. }
$$

Let us introduce a transformation,

$$
u(x, t)=U(\xi) \text { with } \xi=x-c t \text {, where } \mathrm{c} \text { is a parameter. }
$$

With the introduction of the new variable $\xi$, we find the following:

$$
\begin{gathered}
\frac{\partial(. .)}{\partial t}=\frac{\partial \xi}{\partial t} \frac{d(. .)}{d \xi}=-c \frac{d(. .)}{d \xi}, \\
\frac{\partial^{2}(.)}{\partial t^{2}}=c^{2} \frac{\left.d^{2}(.)\right)}{d \xi^{2}} \text { etc. } \\
\frac{\partial(. .)}{\partial x}=\frac{\partial \xi}{\partial x} \frac{d(. .)}{d \xi}=\frac{d(. .)}{d \xi}, \\
\frac{\partial^{2}(. .)}{\partial x^{2}}=\frac{d^{2}(. .)}{d \xi^{2}}, \quad \text { etc. } \\
\frac{\partial^{2}(. .)}{\partial x \partial t}=-c \frac{d^{2}(. .)}{d \xi^{2}} \text { etc. }
\end{gathered}
$$

Using the changes shown in eqns. (3), the NLEE eqn. (1) reduces to a Nonlinear Ordinary Differential Equation (NLODE) of the form

$$
\begin{gathered}
G\left(U, U^{\prime}, U^{\prime \prime}, U^{\prime \prime \prime}, \ldots\right)=0, \\
\text { where } U^{\prime}=\frac{d U}{d \xi}, U^{\prime \prime}=\frac{d^{2} U}{d \xi^{2}}, \text { etc. }
\end{gathered}
$$

If all the terms in the Reduced NLODE eqn. (4) contain derivatives, the equation is to be integrated out as long as all terms contain derivatives and, for simplicity, the integration constants are taken as zero.

Then, we assume the solution of Eqn. (4) in either of the forms 
and

$$
\begin{aligned}
U(\xi) & =\frac{a_{0}}{1+a_{1} \cos \mu \xi} \\
U(\xi) & =\frac{a_{0}}{1+a_{1} \sin \mu \xi}
\end{aligned}
$$

where $a_{0}, a_{1}$ and $\mu$ are parameters to be determined latter.

If we take eqn. (5) as the solution of eqn. (4), we have,

$U^{\prime}=\frac{a_{0} a_{1} \mu \sin \mu \xi}{\left(1+a_{1} \cos \mu \xi\right)^{2}}$,

$$
U^{\prime \prime}=\frac{2 a_{0} a_{1}^{2} \mu^{2}+a_{0} a_{1} \mu^{2} \cos \mu \xi-a_{0} a_{1}^{2} \mu^{2} \cos ^{2} \mu \xi}{\left(1+a_{1} \cos \mu \xi\right)^{3}}, \text { etc. }
$$

If we take eqn. (6) as the solution of eqn. (4), we must have,

$$
\begin{gathered}
U^{\prime}=-\frac{a_{0} a_{1} \mu \cos \mu \xi}{\left(1+a_{1} \sin \mu \xi\right)^{2}}, \\
U^{\prime \prime}=\frac{2 a_{0} a_{1}^{2} \mu^{2}+a_{0} a_{1} \mu^{2} \sin \mu \xi-a_{0} a_{1}^{2} \mu^{2} \sin ^{2} \mu \xi}{\left(1+a_{1} \sin \mu \xi\right)^{3}} \text {, etc. }
\end{gathered}
$$

In one method known as the Rational Cosine Function Method, we substitute eqns. (5) and (7) into the Reduced Nonlinear Ordinary Differential Equation (RNLODE) eqn. (4) and after simplification, we balance suitable exponents of $\cos \mu \xi$. Then, we collect terms with the same power of $\cos \mu \xi$ and the co-efficient is equated to zero. In this way, we obtain a system of algebraic equations among the unknown parameters. Solving such a system of algebraic equations for the unknown parameters and substituting their values into eqn. (5), we obtain the solution of the RNLODE eq. (4) and hence of the given NLEE eqn. (1).

In another method known as the Rational Sine Function Method, we substitute eqns. (6) and (8) into eqn. (4) and after simplification, we balance suitable exponents of $\sin \mu \xi$. Then, we collect terms with the same power of $\sin \mu \xi$ and the co-efficient is equated to zero. Thus, we obtain a system of algebraic equations among the unknown parameters. Solving the system of equations for the unknown parameters and substituting their values into eqn. (6), we will obtain the solution of the RNLODE eqn.(4) and hence of the given NLEE eqn. (1).

\section{Applications}

3.1: Boussinesq Equation: The Boussinesq Equation arises in several physical areas such as in the studies of (i) propagation of long waves in shallow water, (ii) one dimensional nonlinear lattice waves, (iii)vibrations in a nonlinear string, (iv) propagation of ion-acoustic waves in plasmas etc. It reads as

$$
u_{t t}=\alpha u_{x x}+\beta\left(u^{2}\right)_{x x}+\gamma u_{x x x x} \text {, }
$$

where $\alpha, \beta$ and $\gamma$ are real constants.

Then, putting $\xi=x-c t$ ( where $\mathrm{c}$ is a parameter to be determined latter), $u(x, t)=U(\xi)$ and using the changes shown in eqns. (3),we write,

$$
c^{2} U^{\prime \prime}-\alpha U^{\prime \prime}-\beta\left(U^{2}\right)^{\prime \prime}-\gamma U^{\prime \prime \prime \prime}=0 \text {, }
$$

where the primes denote differentiations with respect to $\xi$.

Integrating the above equation twice with respect to $\xi$ and taking the integration constants as zero, we obtain,

$$
\begin{aligned}
& \gamma U^{\prime \prime}+\left(\alpha-c^{2}\right) U+\beta U^{2}=0 \\
& \quad \text { Substituting eqns (5) and (7) into eqn. (10), we obtain, } \\
& \quad 2 \gamma a_{0} a_{1}^{2} \mu^{2}+\gamma a_{0} a_{1} \mu^{2} \cos \mu \xi-\gamma a_{0} a_{1}^{2} \mu^{2} \cos ^{2} \mu \xi+\left(\alpha-c^{2}\right) a_{0} \\
& +2\left(\alpha-c^{2}\right) a_{0} a_{1} \cos \mu \xi+\left(\alpha-c^{2}\right) a_{0} a_{1}^{2} \cos ^{2} \mu \xi+\beta a_{0}^{2}+\beta a_{0}^{2} a_{1} \cos \mu \xi=0
\end{aligned}
$$

For eqn. (11) to be valid, we must have,

$$
\begin{gathered}
2 \gamma a_{1}^{2} \mu^{2}+\left(\alpha-c^{2}\right)+\beta a_{0}=0, \\
\gamma \mu^{2}+2\left(\alpha-c^{2}\right)+\beta a_{0}=0, \\
\left(\alpha-c^{2}\right)-\gamma \mu^{2}=0 .
\end{gathered}
$$

Solving the above system of equations, we obtain,

$$
\begin{aligned}
c & = \pm \sqrt{\alpha-\gamma \mu^{2}}, \\
a_{0} & =-\frac{3 \gamma \mu^{2}}{\beta}, \\
a_{1} & = \pm 1 .
\end{aligned}
$$

Now, substituting eqns. (13) into eqn. (5) and remembering that $\xi=x$-ct, we obtain the solution of the NLEE eqn. (9) as

$$
u(x, t)=-\frac{3 \gamma \mu^{2}}{\beta\left[1 \pm \cos \mu\left\{x \mp\left(\sqrt{\alpha-\gamma \mu^{2}}\right) t\right\}\right]} .
$$

For $\mu=1$, we have the solution, 


$$
u_{1}(x, t)=-\frac{3 \gamma}{\beta[1 \pm \cos \{x \mp(\sqrt{\alpha-\gamma}) t\}]} .
$$

3.2: Gardner Equation:The Gardner Equation, also known as the mixed kdV- mkdV equation, arises in various areas of Nonlinear Physics that includes Fluid Dynamics, Plasma Physics, Quantum Field Theory (QFT), Solid State Physics and many more. It reads as

$$
\begin{aligned}
& u_{t}=\alpha u u_{x}+\beta u^{2} u_{x}+\gamma u_{x x x}, \\
& \text { where } \alpha, \beta \text { and } \gamma \text { are real constants. } \\
& \text { Putting } \xi=x-c t, u(x, t)=U(\xi) \text { and using the changes shown in eqns. (3) as before, we write, } \\
& -c U^{\prime}=\alpha U U^{\prime}+\beta U^{2} U^{\prime}+\gamma U^{\prime \prime \prime} .
\end{aligned}
$$

Integrating once with respect to $\xi$ and taking the integration constant as zero, we have,

$$
\gamma U^{\prime \prime}+c U+\frac{\alpha}{2} U^{2}+\frac{\beta}{3} U^{3}=0 .
$$

Substituting eqns. (5) and (7) into eqn. (16), we obtain,

$$
\begin{aligned}
& 2 \gamma a_{1}^{2} \mu^{2}+\gamma a_{1} \mu^{2} \cos \mu \xi-\gamma a_{1}^{2} \mu^{2} \cos ^{2} \mu \xi+c+2 c a_{1} \cos \mu \xi+c a_{1}^{2} \cos ^{2} \mu \xi \\
& +\frac{\alpha}{2} a_{0}+\frac{\alpha}{2} a_{0} a_{1} \cos \mu \xi+\frac{\beta}{3} a_{0}^{2}=0 .
\end{aligned}
$$

For equation (17) to be valid, we must have,

$$
\begin{gathered}
2 \gamma a_{1}^{2} \mu^{2}+c+\frac{\alpha}{2} a_{0}+\frac{\beta}{3} a_{0}^{2}=0, \\
\gamma \mu^{2}+2 c+\frac{\alpha}{2} a_{0}=0, \\
c-\gamma \mu^{2}=0 .
\end{gathered}
$$

The solutions of the above system of equations are

$$
\begin{aligned}
& \quad c=\gamma \mu^{2}, \\
& a_{0}=-\frac{6 \gamma \mu^{2}}{\alpha}, \\
& a_{1}= \pm \sqrt{1-\frac{6 \beta \gamma \mu^{2}}{\alpha^{2}}} .
\end{aligned}
$$

Now, substituting equations (19) into equation (5) and remembering that $\xi=x-c t$, we obtain the solution of the NLEE eqn. (15) as

$$
u(x, t)=-\frac{6 \gamma \mu^{2}}{\alpha\left[1 \pm\left(\sqrt{1-\frac{6 \beta \gamma \mu^{2}}{\alpha^{2}}}\right) \cos \left(x-\gamma \mu^{2} t\right)\right]} .
$$

For $\mu=1$,we obtain the solution as,

$$
u_{1}(x, t)=-\frac{6 \gamma}{\alpha\left[1 \pm\left(\sqrt{1-\frac{6 \beta \gamma}{\alpha^{2}}}\right) \cos (x-\gamma t)\right]}
$$

3.3: Generalized Boussinesq Burgers Equations: The Generalized Boussinesq- Burgers equations (GBBE) arise in the study of fluid flow and describe the propagation of shallow water waves. These equations read as

$$
\begin{aligned}
& u_{t}+\alpha u u_{x}+\beta v_{x}=0 \\
& v_{t}+\gamma(u v)_{x}+\lambda u_{x x x}=0
\end{aligned}
$$
we obtain,

If we put $u(x, t)=U(\xi), v(x, t)=V(\xi)$ with $\xi=x-c t$ and using thechanges shown in eqns. (3),

$$
\begin{aligned}
& -c U^{\prime}+\left(\frac{\alpha}{2} U^{2}\right)^{\prime}+(\beta V)^{\prime}=0, \\
& -c V^{\prime}+(\gamma U V)^{\prime}+(\lambda U)^{\prime \prime \prime}=0,
\end{aligned}
$$

where the primes denote differentiations with respect to $\xi$.

Integrating eqn. (22a) once with respect to $\xi$ and choosing the integration constant to be zero, we obtain $-c U+\frac{\alpha}{2} U^{2}+\beta V=0$,

giving $V=\frac{1}{\beta}\left(c U-\frac{\alpha}{2} U^{2}\right)$.

obtain

Integrating eqn. (22b) once with respect to $\xi$ and choosing the integration constant to be zero, we

$$
-c V+\gamma U V+\lambda U^{\prime \prime}=0 \text {. }
$$

Substituting this value of $\mathrm{V}$ into eqn.(24), we obtain,

$$
2 \beta \lambda U^{\prime \prime}-2 c^{2} U+(\alpha+2 \gamma) c U^{2}-\alpha \gamma U^{3}=0 .
$$

Then, substituting eqns. (5) and (7) into eqn. (25), we obtain,

$$
2 \beta \lambda\left(2 a_{0} a_{1}^{2} \mu^{2}+a_{0} a_{1} \mu^{2} \cos \mu \xi-a_{0} a_{1}^{2} \mu^{2} \cos ^{2} \mu \xi\right)-2 c^{2} a_{0}\left(1+2 a_{1} \cos \mu \xi+a_{1}^{2} \cos ^{2} \mu \xi\right)
$$




$$
+(\alpha+2 \gamma) c a_{0}^{2}\left(1+a_{1} \cos \mu \xi\right)-\alpha \gamma a_{0}^{3}=0 .
$$

For eqn. (26) to be valid, we must have,

$$
\begin{aligned}
& 4 \beta \lambda a_{1}^{2} \mu^{2}-2 c^{2}+(\alpha+2 \gamma) c a_{0}-\alpha \gamma a_{0}^{2}=0, \\
& 2 \beta \lambda \mu^{2}-4 c^{2}+(\alpha+2 \gamma) c a_{0}=0,
\end{aligned}
$$

$-\beta \lambda \mu^{2}-c^{2}=0$.

The solutions of the above system of equations are

$$
\begin{aligned}
a_{0}= \pm \frac{6 i \mu \sqrt{\beta \lambda}}{(\alpha+2 \gamma)}, \\
a_{1}= \pm \sqrt{1-\frac{9 \alpha \gamma}{(\alpha+2 \gamma)^{2}}} .
\end{aligned}
$$$$
c= \pm i \mu \sqrt{\beta \lambda}
$$

Now, substituting equations (28) into equation (5) and using eq. (23), we obtain the solution of theNLEEs eqns. (21) as

$$
\begin{gathered}
u(x, t)= \pm \frac{6 i \mu \sqrt{\beta \lambda}}{(\alpha+2 \gamma)} \frac{1}{\left[1 \pm\left(\sqrt{\left.1-\frac{9 \alpha \gamma}{(\alpha+2 \gamma)}\right)} \cos \{\mu(x \mp i \mu \sqrt{\beta \lambda} t)\}\right]\right.}(29) \text { and } v(x, t)= \\
\pm \frac{6 i \mu c \sqrt{\beta \lambda}}{\beta(\alpha+2 \gamma)} \frac{1}{\left[1 \pm\left(\sqrt{\left.1-\frac{9 \alpha \gamma}{(\alpha+2 \gamma)}\right)} \cos \{\mu(x \mp i \mu \sqrt{\beta \lambda} t)\}\right]\right.} \\
+\frac{\alpha}{2 \beta} \frac{36 \beta \lambda \mu^{2}}{(\alpha+2 \gamma)^{2}} \frac{1}{\left[1 \pm\left(\sqrt{1-\frac{9 \alpha \gamma}{(\alpha+2 \gamma)}}\right) \cos \{\mu(x \mp i \mu \sqrt{\beta \lambda} t)\}\right]^{2}}
\end{gathered}
$$

where we use $\xi=x-c t$.

3.4: Mikhailov-ShabatEquations:TheMikhailov-Shabat Equations (MSE)appear in the classification of Integrable Systems and read as

$$
\begin{gathered}
p_{t}=p_{x x}+(p+q) q_{x}-\frac{1}{6}(p+q)^{3}, \\
-q_{t}=q_{x x}-(p+q) p_{x}-\frac{1}{6}(p+q)^{3} .
\end{gathered}
$$

Introducing the transformations

$$
\begin{aligned}
& u(x, t)= \\
& p(x, t)+q(x, t) \\
& q_{x}(x, t)-p_{x}(x, t), \\
& \text { the MS system }(31) \text { becomes } \\
& \quad u_{t}+v_{x}-u u_{x}=0, \\
& \quad v_{t}+(u v)_{x}-u^{2} u_{x}+u_{x x x}=0 .
\end{aligned}
$$$$
(32 a) \text { and } \quad v(x, t)=
$$

Substituting $u(x, t)=U(\xi), v(x, t)=V(\xi)$ with $x-c t$ into eqns. (33)and integrating with respect to $\xi$ with the integration constant taken as zero, we obtain

$$
\begin{aligned}
& V=c U+\frac{1}{2} U^{2}(34) \\
& \text { and }-c V+U V-\frac{1}{3} U^{3}+U^{\prime \prime}=0 .
\end{aligned}
$$

Substituting eqn. (34) into eqn. (35), we obtain

$$
U^{\prime \prime}-c^{2} U+\frac{c}{2} U^{2}+\frac{1}{6} U^{3}=0
$$

Let us assume eqn. (5) as the solution of eqn (36).

Substituting eqns. (5) and (7) into eqn. (36), we obtain

$$
\begin{aligned}
& 2 a_{o} a_{1}^{2} \mu^{2}+a_{0} a_{1} \mu^{2} \cos \mu \xi-a_{o} a_{1}^{2} \mu^{2} \cos ^{2} \mu \xi-c^{2} a_{0}\left(1+2 a_{1} \cos \mu \xi+a_{1}^{2} \cos ^{2} \mu \xi\right) \\
& +\frac{c}{2} a_{0}^{2}\left(1+a_{1} \cos \mu \xi\right)+\frac{1}{6} a_{0}^{3}=0 . \\
& \text { For eqn. (37) to be valid, we must have } \\
& 2 a_{1}^{2} \mu^{2}-c^{2}+\frac{c}{2} a_{0}+\frac{1}{6} a_{0}^{2}=0, \\
& \mu^{2}-2 c^{2}+\frac{c}{2} a_{0}=0 \\
& \quad \text { and } \mu^{2}+c^{2}=0 .
\end{aligned}
$$

The solutions of the above system of equations are

$$
c= \pm i \mu \text {, }
$$




$$
\begin{aligned}
& a_{0}= \pm 6 i \mu, \\
& a_{1}= \pm 2 .
\end{aligned}
$$

Substituting eqns. (39) into eqn. (5), we obtain the solution of eqn. (36) as

$$
U(\xi)= \pm \frac{6 i \mu}{1 \pm 2 \cos \mu \xi}
$$

Substituting eqn. (40) into eqn (34), we obtain

$$
V(\xi)=-\frac{6 \mu^{2}}{1 \pm 2 \cos \mu \xi}-\frac{18 \mu^{2}}{(1 \pm 2 \cos \mu \xi)^{2}} \text {. }
$$

Remembering that $u(x, t)=U(\xi), v(x, t)=V(\xi)$ and $\xi=x-c t$, we have,

$$
\begin{gathered}
u(x, t)= \pm \frac{6 i \mu}{1 \pm 2 \cos \mu(x \mp i \mu t)}, \\
v(x, t)=-\frac{6 \mu^{2}}{1 \pm 2 \cos \mu(x \mp i \mu t)} \\
-\frac{18 \mu^{2}}{(1 \pm 2 \cos \mu(x \mp i \mu t))^{2}} .
\end{gathered}
$$

Now, using eqns. (32), eqn. (42) and eqn. (43); we obtain,

$$
\begin{aligned}
& p_{x}(x, t)=\frac{1}{2}\left[u_{x}(x, t)-v(x . t)\right], \\
& q_{x}(x, t)=\frac{1}{2}\left[u_{x}(x, t)+v(x . t)\right]
\end{aligned}
$$

Integrating equations (44) and (45) partially with respect to $x$ and choosing the integration constants as zero, we obtain,

$$
\begin{array}{r}
p(x, t)=\frac{1}{2} u(x, t)-\frac{1}{2} \int v(x, t) \partial x, \\
q(x, t)=\frac{1}{2} u(x, t)+\frac{1}{2} \int v(x, t) \partial x
\end{array}
$$

Considering only the positive value of $\mathrm{a}_{1}(=+2)$ and using eqns (42), (43), (46) and (47), we obtain

$$
\begin{aligned}
& p(x, t)= \pm \frac{3 i \mu}{1+2 \cos \mu(x \mp i \mu t)}+\mu \sqrt{3} \ln \frac{\sqrt{3}+\tan \left\{\frac{\mu(x \mp i \mu t)}{2}\right\}}{\sqrt{3}-\tan \left\{\frac{\mu(x \mp i \mu t)}{2}\right\}} \\
&-\frac{\mu}{\sqrt{3}} \csc \left[\sec ^{-1}\left\{\frac{1}{\sqrt{3}} \tan \frac{\mu(x \mp i \mu t)}{2}\right\}\right] \cot ^{2}\left[\sec ^{-1}\left\{\frac{1}{\sqrt{3}} \tan \frac{\mu(x \mp i \mu t)}{2}\right\}\right] \\
&-\frac{\mu}{\sqrt{3}} \ln \tan \left[\frac{1}{2} \sec ^{-1}\left\{\frac{1}{\sqrt{3}} \tan \frac{\mu(x \mp i \mu t)}{2}\right\}\right]
\end{aligned}
$$

and $\quad q(x, t)= \pm \frac{3 i \mu}{1+2 \cos \mu(x \mp i \mu t)}-\mu \sqrt{3} \ln \frac{\sqrt{3}+\tan \frac{\mu(x \mp i \mu t)}{2}}{\sqrt{3}-\tan \frac{\mu(x \mp i \mu t)}{2}}$

$$
+\frac{\mu}{\sqrt{3}} \csc \left[\sec ^{-1}\left\{\frac{1}{\sqrt{3}} \tan \frac{\mu(x \mp i \mu t)}{2}\right\}\right]^{2} \cot \left[\sec ^{-1}\left\{\frac{1}{\sqrt{3}} \tan \frac{\mu(x \mp i \mu t)}{2}\right\}\right]
$$

$+\frac{\mu}{\sqrt{3}} \ln \tan \left[\frac{1}{2} \sec ^{-1}\left\{\frac{1}{\sqrt{3}} \tan \frac{\mu(x \mp i \mu t)}{2}\right\}\right]$

For $\mu=1$, we obtain the solutions

$$
\begin{aligned}
p_{1}(x, t)= & \pm \frac{3 i}{1+2 \cos (x \mp i t)}+\sqrt{3} \ln \frac{\sqrt{3}+\tan \frac{(x \mp i t)}{2}}{\sqrt{3}-\tan \frac{(x \mp i t)}{2}} \\
& -\frac{1}{\sqrt{3}} \csc \left[\sec ^{-1}\left\{\frac{1}{\sqrt{3}} \tan \frac{(x \mp i t)}{2}\right\}\right] \cot \left[\sec ^{-1}\left\{\frac{1}{\sqrt{3}} \tan \frac{(x \mp i t)}{2}\right\}\right] \\
- & \frac{1}{\sqrt{3}} \ln \tan \left[\frac{1}{2} \sec ^{-1}\left\{\frac{1}{\sqrt{3}} \tan \frac{(x \mp i t)}{2}\right\}\right] \\
\text { and } q_{1}(x, t)= \pm & \frac{3 i}{1+2 \cos (x \mp i t)}-\mu \sqrt{3} \ln \frac{\sqrt{3}+\tan \frac{(x \mp i t)}{2}}{\sqrt{3}-\tan \frac{(x \mp i t)}{2}} \\
& +\frac{1}{\sqrt{3}} \csc \left[\sec ^{-1}\left\{\frac{1}{\sqrt{3}} \tan \frac{(x \mp i t)}{2}\right\}\right] \cot \left[\sec ^{-1}\left\{\frac{1}{\sqrt{3}} \tan \frac{(x \mp i t)}{2}\right\}\right] \\
+ & \frac{1}{\sqrt{3}} \ln \tan \left[\frac{1}{2} \sec ^{-1}\left\{\frac{1}{\sqrt{3}} \tan \frac{(x \mp i t)}{2}\right\}\right]
\end{aligned}
$$

In similar manners, using eqn. (5) as the assumed solution of a NLEE and finding the values of the parameters involved, we can find the solution of the equations concerned. 


\section{Conclusion}

In this paper, the rational sine-cosine function method has been successfully applied in finding solitary wave solutions to fourNLEEs such as Boussinesq Equation (BE), Gardner Equation (GE), Generalized Boussinesq- Burgers equations (GBBE) and Mikhailov-Shabat Equations (MSE). The method can be extended to solve many other NLEEs arising in the study of solitons and other related areas as well.

\section{References}

[1] Marwan Alquran, Kamel Al-Khaled and HasanAnabeh, New Soliton Solutions for Systems of Nonlinear Evolution Equations by the Rational Sine-Cosine Method, Stud. Math.Sci. 3(1)(2011) $1-9$.

[2] HasibunNaher and Farah Aini Abdullah, The basic $\left(\mathrm{G}^{\prime} / \mathrm{G}\right)$ Expansion Method for the Fourth Order Boussinesq Equation, Applied Mathematics, 3(10)(2012), $1144-1152$.

[3] J. L. Bona, M. Chen and J. C. Saut,Boussinesq Equation and other Systems for Small Amplitude Long Waves in Nonlinear Dispersive Media I: Derivation and Linear Theory. J. Nonlinear Sci. 12 (2002) $283-318$

[4] M.A. Abdel - Razek, A. K. Seddeck and Nassar H. Abdel All: New Exact Jacobi Elliptic Function Solutions for Nonlinear Equations using F-expansion method, Stud.Math.Sci..2(1)(2011) 88-95.

[5] Peter A. Clarkson, Rational Solutions of the Boussinesq Equation, ANAL. APPL.6(4)(2008) 349 - 369.

[6] Sh. SadighBehzadi, Numerical Solution of Boussinesq Equation using Modified AdomianDecomposition and Homotopy Analysis Methods, IJM2C (01) (01)(2011)45 - 58.

[7] TianBao Dan, Qiu Yan-Hong and Chen Ning, Exact Solutions for a class of BoussinesqEquation, Appl. Math. Sci.3 (6) (2009) 257 -265 .

[8] Bin Zheng, Travelling Wave Solutions For Some Nonlinear Evolution Equations By The First Integral Method, WSEAS TRANSACTIONS on MATHEMATICS 10 (8) (1011) 249- 258

[9] Mohammad Najafi, MaliheNajafi and SomayeArbabi, Application of the He's Semi-Inverse Method for Solving the Gardner Equation, Int.J. Modern Math. Sci. 5(2)(2013)59- 66.

[10] Mohammad Najafi, MaliheNajafi and SomayeArbabi, New Exact Traveling Wave Solutions of Gardner Equation, Int. J. Modern App. Physics 2(1) (2013) 34-47.

[11] Samir Hamdi, Brian Morse, Bernard Halphen and William Schiesser, Conservation lawsand invariants of motion for nonlinear internal waves: part II, Nat. Hazards 57 (2011) 609 - 616.

[12] V.M. Vassilev, P.A. Djondjorov, M. Ts. Hadzhilazova and I. M. Mladenov, Traveling Wave Solutions of the Gardner Equation and Motion of Plane Curves Governed by the mKdVFlow,in Applications of Mathematics in Technical and Natural Sciences, AIP Conf. Proc. 1404 (2011) 86 - 93.

[13] Isaiah Elvis Mhlanga and ChaudryMasoodKhalique, Exact Solutions of Generalized Boussinesq-Burgers Equations and (2 +1)dimensional Davey-Stewartson Equations, Journal of Applied Mathematics (2012) 1-8.

[14] Sirendaoreji, NEW EXACT TRAVELLING WAVE SOLUTIONS TO THREE NONLINEAR EVOLUTION EQUATIONS, Appl.Math. J.ChineseUniv.Ser. B .19(2)(2004) 178-186. 